\title{
Enhanced conversion efficiency for harmonic generation with double resonance
}

\author{
Z. Y. Ou* and H. J. Kimble \\ Norman Bridge Laboratory of Physics 12-33, California Institute of Technology, Pasadena, California 91125
}

Received February 4, 1993

\begin{abstract}
Conversion efficiency for $\mathrm{cw}$ harmonic generation is calculated for the situation in which both fundamental and harmonic waves are resonant. Compared with the situation of a singly resonant cavity at the fundamental, the doubly resonant geometry can lead to an increase of the effective nonlinear coefficient. High conversion efficiency can thus be achieved with nonlinear crystals of relatively low nonlinear coefficients and with modest pump power for the fundamental input.
\end{abstract}

The idea of using a resonant cavity to enhance conversion efficiency in $\mathrm{cw}$ second-harmonic generation was first discussed by Ashkin et al., ${ }^{1}$ who found that the conversion efficiency can be greatly increased by resonating either the fundamental or the harmonic field. Because of its simplicity, the scheme of employing a resonator to build up the fundamental power is the one most widely used in practice and has recently led to the achievement of high $\mathrm{cw}$ conversion efficiency. ${ }^{2-7}$ For such a scheme, the conversion efficiency $\eta$ for the ratio of generated second-harmonic power $P_{2, \text { out }}$ to the injected fundamental power $P_{1, \text { in }}$ is found from the relation ${ }^{2,4,7}$

$$
\sqrt{\eta}\left(T_{1}+L_{1}+\sqrt{\eta P_{1, \text { in }} E_{\mathrm{NL}}}\right)^{2}=4 T_{1} \sqrt{P_{1, \mathrm{in}} E_{\mathrm{NL}}},
$$

where $T_{1}$ is the transmission coefficient for the input coupler of the buildup cavity for the fundamental field and $L_{1}$ is the total round-trip linear loss exclusive of $T_{1}$ for this field and where we have assumed that $\left(T_{1}, L_{1}\right) \ll 1 . \quad E_{\mathrm{NL}}$ is the single-pass nonlinear conversion efficiency. Given the values of $L_{1}, P_{1, \text { in }}$, and $E_{\mathrm{NL}}$, one can optimize the transmission of the input coupler $T_{1}$ for impedance matching of the input ${ }^{2,4}$ to find the optimum conversion efficiency $\eta^{\mathrm{op}}$, with

$$
\eta^{\mathrm{op}}=\frac{4 \epsilon_{\mathrm{NL}} P_{1, \mathrm{in}}}{\left(1+\sqrt{1+4 \epsilon_{\mathrm{NL}} P_{1, \mathrm{in}}}\right)^{2}},
$$

where $\epsilon_{\mathrm{NL}} \equiv E_{\mathrm{NL}} / L_{1}{ }^{2}$ provides a figure of merit for singly resonant intracavity harmonic generation. From this expression, it is clear that high conversion efficiency requires that $4 \epsilon_{\mathrm{NL}} P_{1, \text { in }} \gg 1$ or $E_{\mathrm{NL}} P_{1, \text { in }} \gg\left(L_{1} / 2\right)^{2}$, i.e., that the nonlinear conversion be much bigger than the linear loss. For fixed linear loss $L_{1}$, we can achieve this end by increasing either $E_{\mathrm{NL}}$ or $P_{1, \text { in }}$. Unfortunately, the latter option is usually accompanied by unwanted thermal effects that degrade the performance of the buildup cavity so that Eq. (2) breaks down before $\eta^{\text {op }}$ is reached.,6 Alternatively, the available fundamental power $P_{1, \text { in }}$ may be too small for efficient harmonic generation in some applications.

Of course, the option of increasing the material nonlinearity as expressed by $E_{\mathrm{NL}}$ (relative to $L_{1}$ ) is clearly the pathway of choice and has been followed in the experiments that have achieved high conversion efficiency with crystals with large nonlinear coefficients, such as $\mathrm{LiNbO}_{3}, \mathrm{KNbO}_{3}$, and $\mathrm{KTP} .^{2-7}$ However, not withstanding some promising new methods, ${ }^{6}$ crystals with large $E_{\mathrm{NL}}$ are available only for limited regions in wavelength. Faced with this state of affairs, we describe in this Letter a technique for enhancing $E_{\mathrm{NL}}$ by resonating the harmonic as well as the fundamental field. Although Ashkin et al. ${ }^{1}$ did discuss the possibility of using a harmonic resonator to enhance $E_{\mathrm{NL}}$, they did not pursue the scheme of resonating both fundamental and harmonic waves, perhaps because of the associated technical complexity.

As the starting point in our analysis, we consider the situation where both the fundamental and the harmonic fields are separately resonant, either in two independent but overlapping cavities (as shown in Fig. 1) or in the same physical cavity (not shown) with high finesse at both the fundamental and harmonic frequencies. The transmission coefficients for input and output couplers of the two cavities are denoted by $T_{1}$ and $T_{2}$, respectively. $L_{k}$ is the total round-trip linear loss exclusive of $T_{k}$ in the cavity $k$, whereas $d_{k}$ is the effective cavity round-trip length. $k=$ 1,2 corresponds to the fundamental and harmonic fields of frequencies $\omega_{1}$ and $\omega_{2}=2 \omega_{1}$, respectively. By assuming that the fields in the two cavities are coupled through a nonlinear crystal with coupling constant $\kappa$, we find that the equations describing the coupled system at steady state are given by ${ }^{8}$

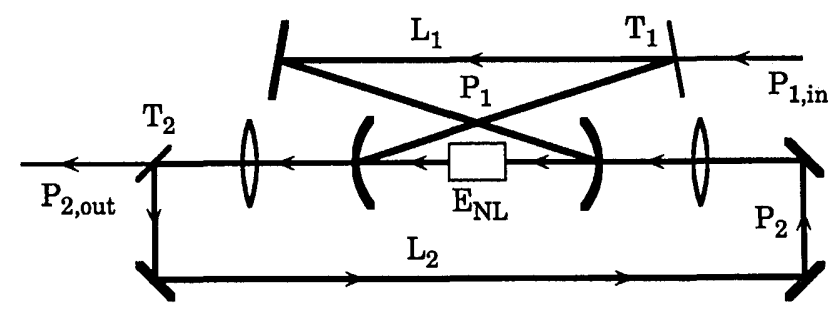

Fig. 1. Geometry of doubly resonant scheme for harmonic generation. 


$$
\begin{aligned}
-\Gamma_{1} \alpha_{1}+\kappa^{*} \alpha_{1}{ }^{*} \alpha_{2}+\epsilon_{1} & =0 \\
-\Gamma_{2} \alpha_{2}-\frac{1}{2} \kappa \alpha_{1}^{2} & =0
\end{aligned}
$$

Here the cavity decay rate $\Gamma_{k}=c \pi / d_{k} F_{k}$, with the cavity finesse $F_{k} \equiv 2 \pi /\left(T_{k}+L_{k}\right)$. The coupling coefficient $\kappa$ can be expressed in terms of the single-pass nonlinear conversion efficiency $E_{\mathrm{NL}}$ by $^{9}$

$$
|\kappa|^{2}=E_{\mathrm{NL}} \frac{4 \pi \hbar c^{4}}{d_{1}^{2} d_{2} \lambda_{1}}
$$

$\alpha_{k}$ is the amplitude of the field in cavity $k$ and is related to the intracavity power $P_{k}$ by

$$
P_{k}=\frac{c \hbar \omega_{k}}{d_{k}}\left|\alpha_{k}\right|^{2} \quad(k=1,2) .
$$

In Eqs. (3), $\epsilon_{1}$ is connected to the input power $P_{1, \text { in }}$ by

$$
T_{1} P_{1, \text { in }}=\left|\epsilon_{1}\right|^{2} \hbar \omega_{1} d_{1} / c
$$

and the output power $P_{2, \text { out }}$ is simply related to $P_{2}$ by

$$
P_{2, \text { out }}=T_{2} P_{2} \text {. }
$$

From the above, we can find the overall conversion efficiency $\eta \equiv P_{2, \text { out }} / P_{1, \text { in }}$ from the fundamental input field to the harmonic output field. With a little algebra we have

$$
\begin{aligned}
\sqrt{\eta}\left(T_{1}+L_{1}+\sqrt{4 \eta P_{1, \text { in }} E_{\mathrm{NL}} / T_{2}}\right)^{2} & \\
& =\frac{4 T_{1} T_{2}}{T_{2}+L_{2}} \sqrt{4 P_{1, \text { in }} E_{\mathrm{NL}} / T_{2}},
\end{aligned}
$$

where all the quantities are measurable.

Let us now define a new set of variables that are associated with the doubly resonant situation:

$$
\begin{aligned}
& E_{\mathrm{NL}}^{\mathrm{eff}} \equiv 4 E_{\mathrm{NL}} / T_{2}, \\
& T_{1}^{\mathrm{eff}} \equiv \frac{T_{1} T_{2}}{T_{2}+L_{2}} \equiv \frac{T_{1}}{1+\beta_{2}}, \\
& L_{1}^{\mathrm{eff}} \equiv L_{1}+\frac{T_{1} \beta_{2}}{1+\beta_{2}},
\end{aligned}
$$

with $\beta_{2}=L_{2} / T_{2}$. Equation (8) can then be written as

$$
\sqrt{\eta}\left(T_{1}^{\mathrm{eff}}+L_{1}^{\mathrm{eff}}+\sqrt{\eta P_{1, \mathrm{in}} E_{\mathrm{NL}}^{\mathrm{eff}}}\right)^{2}=4 T_{1}^{\mathrm{eff}} \sqrt{P_{1, \mathrm{in}} E_{\mathrm{NL}}^{\mathrm{eff}}}
$$

which is precisely of the form of Eq. (1) for the conversion efficiency of $\eta$ in the absence of the harmonic cavity. But here the nonlinear coefficient $E_{\mathrm{NL}}$ is enhanced by the factor $4 / T_{2}$ to produce an effective value $E_{\mathrm{NL}}^{\mathrm{eff}}$, which can be understood as an increase of the effective crystal length brought about by the harmonic enhancement cavity.

To illustrate the effectiveness of this technique, we apply it to a specific example of doubling with an LBO crystal, which is not generally an efficient crystal for $\mathrm{cw}$ harmonic generation. To achieve a moderate efficiency for the scheme of resonating only the fundamental, a large input power is required. ${ }^{10}$ However, with the harmonic enhancement cavity, a relatively small input power should be sufficient for the same efficiency, as is demonstrated in Fig. 2, where we plot the efficiency $\eta$ for frequency doubling versus the input power $P_{1, \text { in }}$ for the schemes with [curves (i)-(iii)] and without [curve (iv)] a harmonic enhancement cavity. Different linear losses $L_{2}$ for the harmonic field are assumed in the schemes for curves (i)-(iii), whereas the loss $L_{1}$ of the fundamental field and the value of $E_{\mathrm{NL}}$ are taken from Ref. 10 $\left(E_{\mathrm{NL}}=6 \times 10^{-5} \mathrm{~W}^{-1}, L_{1}=0.017\right)$. The input and output coupling coefficients $T_{1}$ and $T_{2}$ are optimized for best conversion efficiency at input power of $0.3 \mathrm{~W}$. The improvement with the harmonic cavity is clear in this figure.

The increase $E_{\mathrm{NL}} \rightarrow E_{\mathrm{NL}}^{\mathrm{eff}}$ is unfortunately accompanied by an increase in the effective round-trip loss of the fundamental $L_{1} \rightarrow L_{1}^{\text {eff }}$. The increase arises from the passive losses $L_{2}$ of the harmonic cavity as expressed by the ratio $\beta_{2}=L_{2} / T_{2}$. Therefore a trade-off must be made between minimizing $\beta_{2}$ and maximizing $E_{\mathrm{N}}^{\text {eff }}$ in order to optimize the conversion efficiency $\eta$. Given $E_{\mathrm{NL}}, P_{1, \mathrm{i}}, L_{1}$, and $L_{2}$, we find the optimized values of $T_{1}, T_{2}$, and $\eta$ as

$$
\begin{aligned}
\eta^{\mathrm{op}} & =\frac{\bar{P}\left[2\left(s_{+}+s_{-}\right)-1 / 3\right]}{\left(s_{+}+s_{-}+1 / 3\right)^{2}}, \\
& \simeq \frac{T_{2}^{\mathrm{op}}}{T_{2}^{\mathrm{op}}+L_{2}} \frac{T_{1}^{\mathrm{op}}}{T_{1}^{\mathrm{op}}+L_{1}}, \\
\beta_{1}{ }^{-1} & \equiv T_{1}^{\mathrm{op}} / L_{1}=s_{+}+s_{-}+1 / 3, \\
& \simeq(2 \bar{P})^{1 / 3}+1 / 3 \\
\beta_{2}{ }^{-1} & \equiv T_{2}^{\mathrm{op}} / L_{2}=2\left(s_{+}+s_{-}\right)-1 / 3, \\
& \simeq 2(2 \bar{P})^{1 / 3}-1 / 3
\end{aligned}
$$

with

$$
s_{ \pm}=[1 / 27+\bar{P} \pm \sqrt{\bar{P}(\bar{P}+2 / 27)}]^{1 / 3}
$$

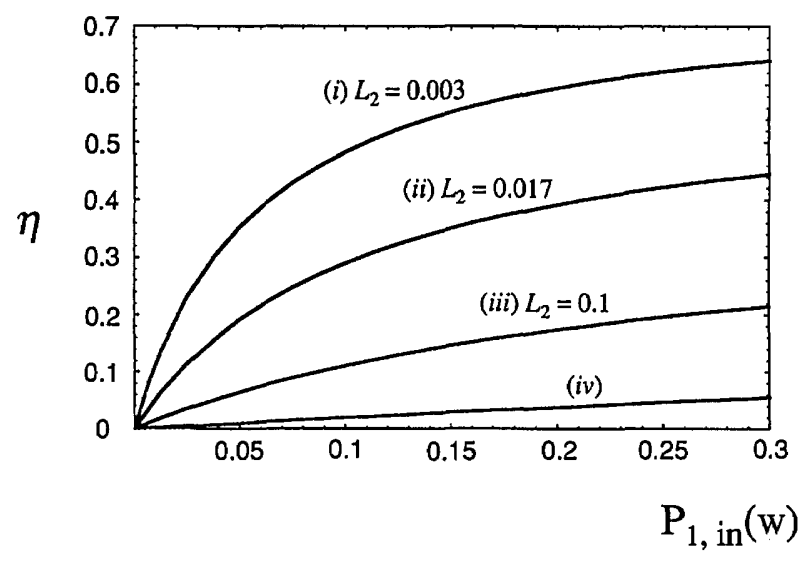

Fig. 2. Harmonic conversion efficiency $\eta$ plotted as a function of input fundamental power $P_{1, \text { in }}$ for doubly [curves (i)-(iii)] and singly [curve (iv)] resonant schemes as derived from Eqs. (9) and (1). The parameters are $E_{\mathrm{NL}}=6 \times 10^{-5} \mathrm{~W}^{-1}, L_{1}=0.017$ for all four schemes, and $L_{2}=0.003, T_{1}=0.064, T_{2}=0.019$ (curve $\mathrm{i}$ ); $L_{2}=0.017$, $T_{1}=0.039, T_{2}=0.06$ (curve ii); $L_{2}=0.1, T_{1}=0.024$, $T_{2}=0.18$ (curve iii); $T_{1}=0.018$ and no harmonic enhancement cavity (curve iv). 
(a)

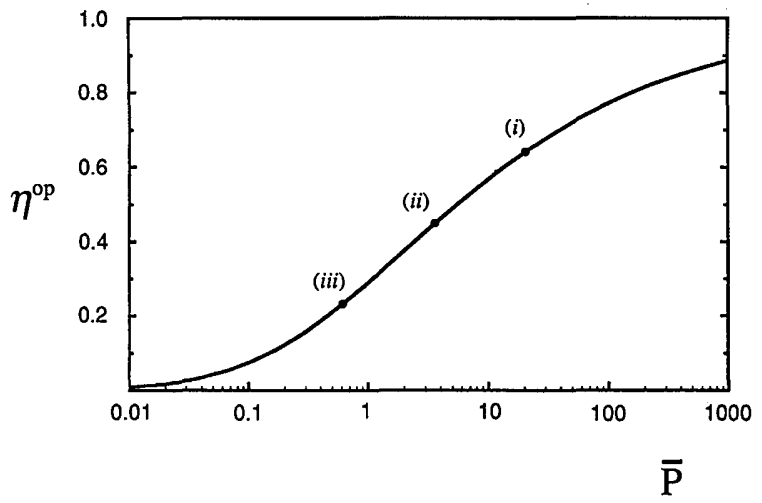

(b)

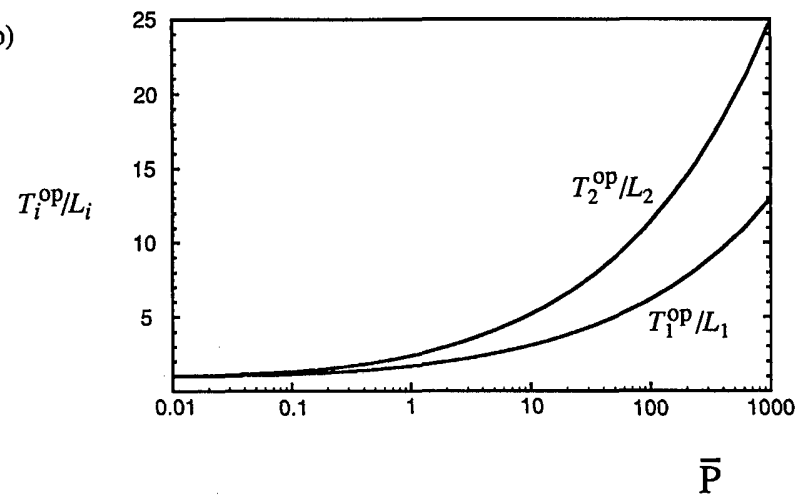

Fig. 3. (a) Optimized conversion efficiency $\eta^{\text {op }}$ and (b) optimized coupling coefficients $T_{1}^{\mathrm{op}} / L_{1}\left(\beta_{1}{ }^{-1}\right), T_{2}^{\mathrm{op}} / L_{2}$ $\left(\beta_{2}{ }^{-1}\right)$ as a function of the dimensionless input power $\bar{P}=E_{\mathrm{NL}} P_{1, \text { in }} / L_{1}^{2} L_{2}$.

Here $\bar{P} \equiv P_{1, \text { in }} \bar{\epsilon}_{N L} \equiv E_{\mathrm{NL}} P_{1, \text { in }} / L_{1}^{2} L_{2}$ is the normalized input fundamental power and $\bar{\epsilon}_{\mathrm{NL}} \equiv E_{\mathrm{NL}} / L_{1}^{2} L_{2}$ provides a figure of merit for doubly resonant intracavity harmonic generation. The assumption $E_{\mathrm{NL}} / P_{1, \text { in }} L_{1} / L_{2} \gg\left(L_{1} / 3\right)^{3}$ (that is, relatively strong input power) is taken in the approximation from relations (11) to (12). In Fig. 3 we plot the optimized conversion efficiency $\eta^{\mathrm{op}}$ as well as $T_{1}^{\mathrm{op}} / L_{1}\left(\equiv \beta_{1}{ }^{-1}\right)$, $T_{2}^{\mathrm{op}} / L_{2}\left(\equiv \beta_{2}{ }^{-1}\right)$ versus the normalized input power $\bar{P}$. The dots in Fig. 3(a) correspond to curves (i)-(iii) in Fig. 2 for $P_{1, \text { in }}=0.3 \mathrm{~W}$. Note that Fig. 3 is a universal curve appropriate to any doubly resonant scheme when the input power is expressed in terms of the dimensionless parameter $\bar{P}$ [a similar universal curve follows form Eq. (2) for $\left.\bar{P}=\epsilon_{\mathrm{NL}} P_{1, \text { in }}\right]$. One caveat is that relations (3)-(12) are correct only in the highfinesse approximation, that is, for $T_{1}, T_{2}, L_{1}, L_{2} \ll 1$. In particular, the limit of $T_{2} \rightarrow 1$ cannot be applied to Eqs. (8) and (9) for the transition to Eq. (2). Of course, a more general formula for the conversion efficiency $\eta$ can be derived for arbitrary $T$ and $L$. Since the derivation is much more complicated, we will present here only the final result for $\eta$, which has the form of

$$
\begin{array}{r}
\left(\frac{\eta}{1-L_{2}}\right)^{1 / 2}\left[\frac{\pi}{F_{1}-\pi}+\left(1-\frac{\pi}{2 F_{2}}\right)\left(\frac{\eta P_{1, \mathrm{in}} E_{\mathrm{NL}}}{T_{2}\left(1-L_{2}\right)}\right)^{1 / 2}\right] \\
=\frac{T_{1} F_{2} \sqrt{P_{1, \mathrm{in}} E_{\mathrm{NL}} T_{2}}}{\pi\left(1-T_{1}\right)},
\end{array}
$$

where $F_{1,2} \equiv\left\{1-\left[\left(1-T_{1,2}\right)\left(1-L_{1,2}\right)\right]^{1 / 2}\right\}$.

In summary, we have calculated the conversion efficiency for harmonic generation with both the fundamental and harmonic waves in resonance. Compared with the scheme of only the fundamental resonance, here the single-pass nonlinear conversion efficiency is increased by a factor of approximately the finesse of the harmonic cavity such that relatively small input power is enough to achieve high conversion efficiency. Because of the deleterious role of losses, the technique should be most powerful for crystals such as $\mathrm{KDP}, \mathrm{LBO}$, and $\mathrm{BBO}$, which have very low losses $L_{1,2}$ and also have relatively small nonlinear coefficients $E_{\mathrm{NL}}$. Since the technique relies on doubly resonant cavities, we caution that it is vulnerable to subtle changes in cavity properties, such as thermal lensing, ${ }^{4,6}$ as well as to some nonlinear loss effects in some crystals. ${ }^{4}$ However, the technique can be made to work in a robust fashion, as demonstrated in Ref. 11, where $4 \mathrm{~mW}$ of harmonic output was generated for only $15 \mathrm{~mW}$ of fundamental input in a doubly resonant cavity. Finally, note that although instability may occur for high input power, as predicted from the time-dependent form of Eqs. (3), ${ }^{8}$ one can always choose the optimized input (output) coupler $T_{1(2)}^{\mathrm{op}}$ to avoid such a case.

This study was supported by the National Science Foundation and the U.S. Office of Naval Research. We thank F. Meiere for assistance in the preparation of the figures.

*Present address, Department of Physics, Indiana University-Purdue University at Indianapolis, Indianapolis, Indiana 46202.

\section{References}

1. A. Ashkin, G. D. Boyd, and J. M. Dziedzic, IEEE J. Quantum Electron. QE-2, 109 (1966).

2. W. J. Kozlovsky, C. D. Nabors, and R. L. Byer, IEEE J. Quantum Electron. 24, 913 (1988).

3. W. J. Kozlovsky, W. Length, E. E. Latta, A. Moser, and G. L. Bona, Appl. Phys. Lett. 56, 2291 (1990).

4. E. S. Polzik and H. J. Kimble, Opt. Lett. 16, 731 (1991); in Digest of Conference on Compact Blue-Green Lasers (Optical Society of America, Washington, D.C., 1992), paper ThD6.

5. D. C. Gerstenberger, G. E. Tye, and R. W. Wallace, Opt. Lett. 16, 992 (1991); in Digest of Conference on Compact Blue-Green Lasers (Optical Society of America, Washington, D.C., 1992), paper FA4.

6. D. H. Jundt, M. M. Fejer, R. L. Byer, R. G. Norwood, and P. F. Bordui, Opt. Lett. 16, 1856 (1991).

7. Z. Y. Ou, S. F. Pereira, E. S. Polzik, and H. J. Kimble, Opt. Lett. 17, 640 (1992).

8. P. D. Drummond, K. J. McNeil, and D. F. Walls, Opt. Acta 27, 321 (1980).

9. L. A. Wu, M. Xiao, and H. J. Kimble, J. Opt. Soc. Am. B 4, 1465 (1987).

10. S. T. Yang, C. C. Pohalski, E. K. Gustafson, R. L. Byer, R. S. Feigelson, R. J. Raymakers, and R. Route, Opt. Lett. 16, 1493 (1991).

11. S. F. Pereira, M. Xiao, H. J. Kimble, and J. L. Hall, Phys. Rev. A 38, 4931 (1989). 